\title{
Datations radiocarbone
}

L'habitat magdalénien de la grotte du Bourrouilla à Arancou (PyrénéesAtlantiques)

Michel Fontugne, Christine Hatté

\section{Citer ce document / Cite this document :}

Fontugne Michel, Hatté Christine. Datations radiocarbone. In: Gallia préhistoire, tome 41, 1999. pp. 10-12;

doi : https://doi.org/10.3406/galip.1999.2339

https://www.persee.fr/doc/galip_0016-4127_1999_num_41_1_2339

Fichier pdf généré le 28/03/2019 
Les diverses catégories de matériel provenant de ces deux opérations sont décrites dans les pages qui suivent par les personnes qui se sont chargées de leur étude ${ }^{1}$. Dès avant la présente publication, le gisement d'Arancou a été l'objet de plusieurs articles scientifiques ou de vulgarisation ainsi que de deux travaux universitaires : Chauchat, 1991 ; Dachary, 1993; Crémadès, 1996a, 1996b, 1997; Fritz, 1995a et b, 1996; Le Gall, Martin, 1996 ; Roussot, 1995-1996. Le gisement et l'art mobilier d'Arancou ont été présentés au séminaire international "Représentations préhistoriques " dirigé par D. Vialou lors de la sćance de févricr 1994, par C. Chauchat, A. Roussot, M. Crémadès et C. Fritz. Enfin, une exposition tenue au musée de Terra Amata à Nice, "Présence animale dans la vie quotidienne des hommes préhistoriques ", a montré des moulages de quelques objets d'Arancou, prêtés grâce à l'amabilité du conservateur du musée national de Préhistoire des Eyzies, J.J. Cleyet-Merle, et le catalogue de l'exposition contient des photographies de ces objets.

C. C.

\section{DATATIONS RADIOCARBONE}

Les datations carbone 14 ont été effectuées sur six échantillons d'ossements provenant des couches B2, B3, $\mathrm{C} 1, \mathrm{C} 2$ et C4. Malgré la faible quantité d'ossements $(<200 \mathrm{~g})$, leur bonne conservation a permis d'extraire selon une méthode dérivée de Longin en 1971 (Délibrias, 1985) une quantité suffisante de collagène. Les âges radiocarbone ont été obtenus par comptage $B$ (compteurs proportionnels à gaz, $\left.\mathrm{CO}_{2}\right)$ et sont reportés dans le tableau I. Pour obtenir une bonne précision nous

1. Les études spécialiscées qui suivent ont été rédligées par des auteurs de formation, de culture et même d'origine géographique très diverses. Nous avons essayé de faire en sortc que la présentation des données soit la meilleure possible étant domné l'état actuel de nos disciplines, les contraintes de la récolte de ces domnées sur le terrain ct celles imposées par lat place limitée dans une publication. Mais il ta de soi que les interprétations et les appréciations de chaque auteur ne sauraient engager les autres, et en particulier le directeur ou coordinateur de cette publication collective. Des discussions ont eu lieu sur quelques points et le lecteur doit être laissé libre d'exercer son esprit critique, comme il convient à toute production qui se veut scientifique.
Tabl. I - Dates radiocarbone de la grotte du Bourrouilla à Arancou.

\begin{tabular}{|c|c|c|c|c|}
\hline $\mathbf{N}^{\circ}$ laboratoire & Niveau & Âge conventionnel BP & Dates calibrées & $\delta^{\mathbf{1 3}} \mathbf{C}(\mathbf{0} \mathbf{0 0})$ \\
\hline Gif -9986 & B2 & $12395 \pm 35$ & $14800-14231$ & $-21,42$ \\
\hline Gif -10002 & B2 & $12260 \pm 120$ & $14755-13943$ & $-21,54$ \\
\hline Gif -10234 & C1 & $12700 \pm 40$ & $15252-14622$ & $-20,91$ \\
\hline Gif -10235 & C4 & $12710 \pm 90$ & $15353-14569$ & $-20,82$ \\
\hline Gif -10254 & C2 & $13220 \pm 80$ & $16077-15434$ & $-21,30$ \\
\hline Gif -10255 & B3 & $12780 \pm 40$ & $15371-14741$ & $-21,77$ \\
\hline
\end{tabular}

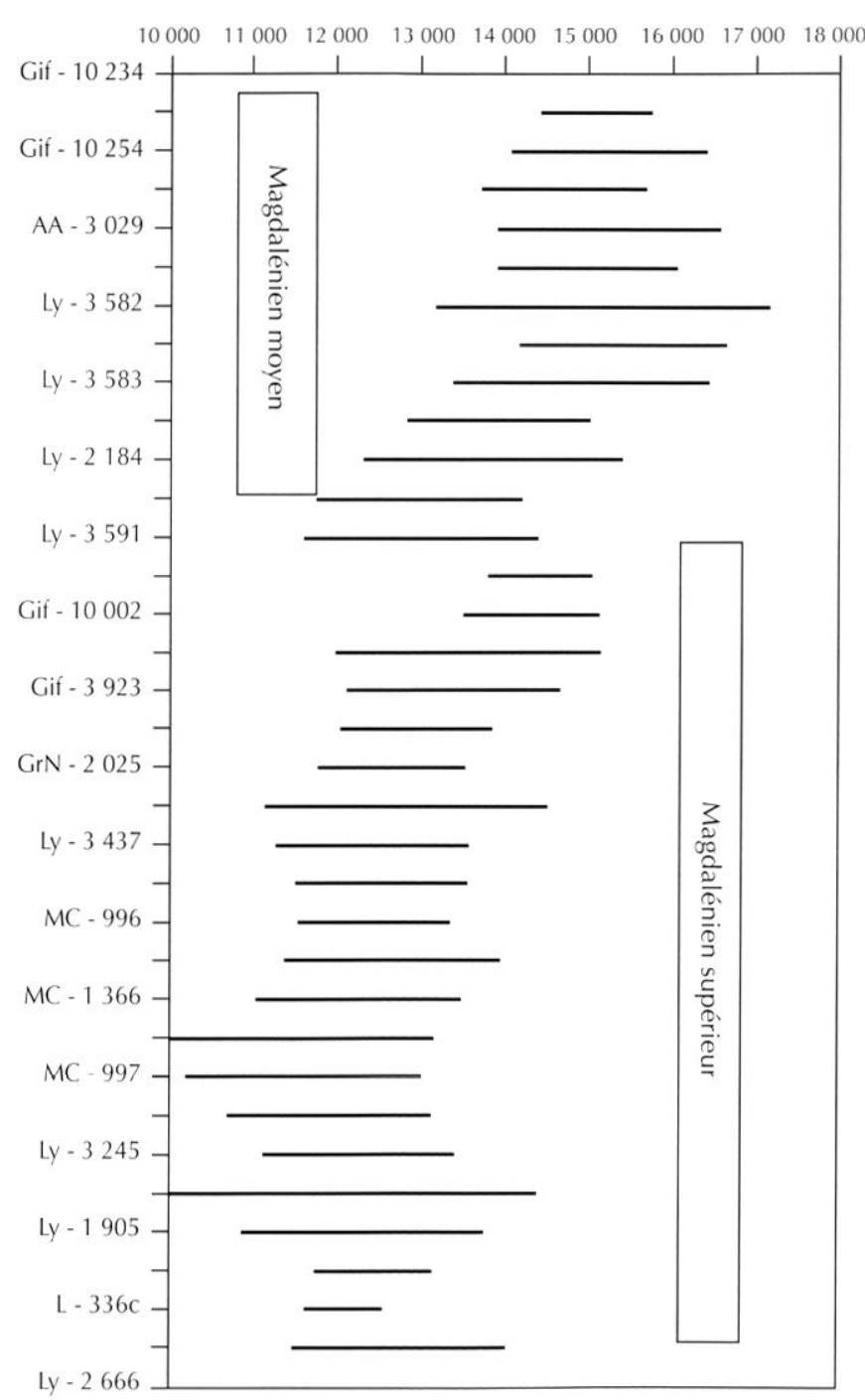

Fig. 6 - Datations radiocarbone calibrées, avant le présent, du Magdalénien moyen et supérieur des Pyrénées occidentales (cf. tabl. II). 
Tabl. II - Dates radiocarbone du Magdalénien moyen et supérieur cies gisements pyrénéens.

\begin{tabular}{|c|c|c|c|c|c|}
\hline$N^{\circ}$ laboratoire & Site & Niveau & Âge conventionnel BP & Dates calibrées BP & Phase culturelle \\
\hline AA -3029 & Dufaure & 6 base & $14640 \pm 230$ & $16087-15042$ & Magdalénien moyen \\
\hline$A A-3030$ & Dufaure & 6 base & $14590 \pm 100$ & $15797-15242$ & Magdalénien moyen \\
\hline Ly - 3582 & Dufaure & 5 base & $14570 \pm 390$ & $16316-14598$ & Magdalénien moyen \\
\hline BM - 2375 & Berroberria & & $14430 \pm 290$ & $15987-14654$ & Magdalénien moyen \\
\hline Ly -3583 & Dufaure & 6 sommet & $14020 \pm 340$ & $15648-13989$ & Magdalénien moyen \\
\hline Ly - 2923 & Dufaure & 5 & $12990 \pm 270$ & $14240-12619$ & Magdalénien moyen \\
\hline Ly - 2184 & Fontanet & surface & $12770 \pm 420$ & $14292-12012$ & Magdalénien moyen \\
\hline Gif - 2943 & Le Portel & & $12760 \pm 170$ & $13670-12529$ & Magdalénien moyen \\
\hline Ly - 3591 & Dufaure & 5 & $12690 \pm 230$ & $13721-12310$ & Magdalénien moyen \\
\hline BM - 2372 & Berroberria & & $13270 \pm 220$ & $14506-13173$ & Magdalénien supérieur \\
\hline Gif - 3923 & Églises & 8bis & $12900 \pm 220$ & $13998-12611$ & Magdalénien supérieur \\
\hline GrN - 2026 & La Vache & Monique 4 & $12850 \pm 60$ & $13555-12879$ & Magdalénien supérieur \\
\hline GrN - 2025 & La Vache & Monique 2 & $12540 \pm 105$ & $13183-12365$ & Magdalénien supérieur \\
\hline Ly - 1906 & Espelugues & Calvaire $6 \mathrm{~m}$ & $12450 \pm 330$ & $13635-11805$ & Magdalénien supérieur \\
\hline Ly -3437 & Lortet & & $12300 \pm 200$ & $13055-11887$ & Magdalénien supérieur \\
\hline I- 13439 & Erralla & III & $12310 \pm 190$ & 13042 - 11921 & Magdalénien supérieur \\
\hline MC - 996 & Rhodes II & F5 sommet & $12300 \pm 150$ & $12931-11985$ & Magdalénien supérieur \\
\hline Gif - 2950 & Belvis & 3 & $12270 \pm 280$ & $13225-11695$ & Magdalénien supérieur \\
\hline$M C-1366$ & Rhodes II & F5 & $12250 \pm 200$ & $12977-11832$ & Magdalénien supérieur \\
\hline Gif -2258 & Rhodes II & F5 & $12160 \pm 160$ & $12751-11804$ & Magdalénien supérieur \\
\hline MC - 997 & Rhodes II & F6 base & $12100 \pm 150$ & $12641-11753$ & Magdalénien supérieur \\
\hline I - 9240 & Ekaïn & VI base & $12050 \pm 190$ & $12665-11625$ & Magdalénien supérieur \\
\hline Ly - 3245 & Dufaure & 4 & $12030 \pm 260$ & $12807-11470$ & Magdalénien supérieur \\
\hline Gif - 1434 & Églises & 8 & $11800 \pm 500$ & $13226-10749$ & Magdalénien supérieur \\
\hline Ly - 1905 & Espelugues & calvaire $1-2 \mathrm{~m}$ & $11750 \pm 430$ & $12860-10877$ & Magdalénien supérieur \\
\hline Ly - 3181 & Dufaure & 4 & $11750 \pm 300$ & $12511-11112$ & Magdalénien supérieur \\
\hline$L-336 c$ & La Vache & Monique 2 & $11650 \pm 200$ & $12158-11194$ & Magdalénien supérieur \\
\hline M - 1023 & Carreres & & $11450 \pm 500$ & $12815-10255$ & Magdalénien supérieur \\
\hline Ly - 2666 & Dufaure & Sommet 4 & $10910 \pm 220$ & $11342-10396$ & Magdalénien supérieur \\
\hline
\end{tabular}


avons effectué la plupart des comptages au laboratoire souterrain de Modane (Savoie). Ce laboratoire situé à $1750 \mathrm{~m}$ sous le sommet du mont Fréjus, à l'abri du rayonnement cosmique, permet l'utilisation de compteurs de grand volume avec un très bas bruit de fond (Fontugne et al., 1994). Les dates sont corrigées du fractionnement biologique (correction de $\delta^{13} \mathrm{C}$, cf. Stuiver, Polach, 1977) ; les rapports isotopiques du carbone entre - 20 et - $22 \%$ pour le collagène sont comparables à ceux obtenus pour la plupart des gisements du Paléolithique. Les intervalles de dates calibrées ont été calculés grâce au logiciel de Stuiver et Reimer (1993). Malgré la bonne précision obtenue pour ces datations, il faut remarquer que les intervalles de dates calibrées (95\% de confiance ou 2 sigma) sont de l'ordre de 660 ans en moyenne, donc inférieurs d'un facteur 2 à 3 par rapport à ceux obtenus pour le Magdalénien du site voisin de l'abri Dufaure à Sorde-l'Abbaye (Landes) ou d'autres sites pyrénéens (tabl. II).

Les résultats de la grotte du Bourrouilla sont relativement homogènes : la couche $B 2$ (Magdalénien supérieur) autour de 12300 ans BP, les couches B3-C1C4 (Magdalénien moyen) autour de 12700 ans BP. Seule la date (Gif-10254) de la couche C2 n'est pas en accord avec la stratigraphie, elle semble trop vieille et est incompatible avec celles des couches voisines supposées contemporaines. La première explication pourrait être une contamination par du matériel plus ancien qui n'aurait pas été détecté. Cette interprétation reste plausible compte tenu de la légère pente des couches observée lors du sondage et du grand nombre de carrés échantillonnés pour réunir suffisamment de matériel pour la datation.

Les âges obtenus pour le Magdalénien supérieur sont en parfait accord avec ceux obtenus par d'autres auteurs dans les Pyrénées françaises ou espagnoles ( $c f$. la synthèse de Straus, Évin, 1989 et Évin, 1995). Il en est de même pour le Magdalénien moyen, ce qui n'est pas étonnant si l'on considère la dispersion des âges enregistrée pour cette phase culturelle notamment à l'abri Dufaure (Straus, Évin, 1989) qui n'est pas très éloigné d'Arancou (fig. 6 et tabl. II). Cependant, les dates obtenues n'atteignent jamais 14000 ans BP.

En conclusion, la datation des restes osseux de la grotte du Bourrouilla permet de mettre en évidence deux phases : l'une autour de 12300 ans BP associée au Magdalénien supérieur, l'autre au Magdalénien moyen vers 12700 ans BP. Ces datations apportent une contribution nouvelle à la chronologie du Magdalénien des Pyrénées qui reste néanmoins imprécise si l'on considère la dispersion des datations.

M. F. et C. H.

\section{L'INDUSTRIE LITHIQUE}

L'industrie lithique de la grotte du Bourrouilla, dont nous présentons une première étude ici, se subdivise en deux ensembles définis selon l'historique décrit ci-dessus. Le premier regroupe les pièces découvertes et cotées lors du sondage de 1990. Le second est constitué de l'outillage récolté dans les déblais de la fouille clandestine.

Le contexte de la découverte conduit à aborder ces deux ensembles différemment.

Le premier, étudié de manière approfondie, apporte des données capitales tant du point de vue de la matière première utilisée que du point de vue de la production et de l'utilisation des supports d'outils. Les refus de tamis ont été consultés et quelques pièces extraites lorsqu'elles apportaient un complément d'information.

Le second, étudié globalement, fournit des informations d'ordre surtout qualitatif : données techniques complémentaires, présence ou absence d'outils attribués au Magdalénien, présence ou absence d'outils révélant des occupations d'autres périodes.

\section{PREMIER ENSEMBLE : MATÉRIEL DU SONDAGE}

À quels faciès technoculturels correspondent les divers niveaux archéologiques définis à la fouille (fig. 7 à 9) ? Est-il possible de reconstituer la ou les chaines opératoires de fabrication des supports d'outil ? Quelle est la place des supports transformés dans ces chaînes opératoires? Quels sont les outils abandonnés sur le site ? Estil possible de distinguer différents gîtes d'approvisionnement en matières premières?

Avant de répondre à ces diverses interrogations, il nous a semblé indispensable de :

- tenter d'évaluer l'état de préservation des niveaux archéologiques; en effet, les fouilles ont mis en évidence l'existence de bioturbations ; 